DOI: https://doi.org/10.46296/yc.v4i6edesp.0030

\title{
CONSTRUCCIÓN DE UN SISTEMA DE BAJO COSTO PARA LA MEDICIÓN DE RAYOS ULTRAVIOLETA BASADO EN INTERNET DE LAS COSAS
}

\section{CONSTRUCTION OF A LOW-COST SYSTEM FOR BASED MEASUREMENT OF ULTRAVIOLET RAYS ON THE INTERNET OF THINGS}

\author{
Verduga-Urdánigo Fabricio ${ }^{1 *}$; Cevallos-Macías John²; Solórzano-Cadena Rubén ${ }^{3}$ \\ ${ }^{1}$ Universidad Internacional de la Rioja UNIR. Logroño, España. \\ ¿Universidad Laica "Eloy Alfaro" de Manabí. Manta, Ecuador. \\ "Universidad Laica "Eloy Alfaro" de Manabí. Manta, Ecuador.
}

*Correo: frankyfa@hotmail.com

\begin{abstract}
Resumen
El presente trabajo de investigación publica la creación y evaluación de un sistema de bajo costo y con acceso a Internet para la medición de la radiación ultravioleta UV-B. El sistema desarrollado utiliza componentes de hardware como placas Arduino, Raspberry Pi, sensores de luz, sensor UV; y programación de software en lenguajes como C++, Python, HTML y JavaScript. El hardware cuenta también con un dispositivo de conexión inalámbrica que facilita conectarse al Internet para poder publicar los datos recopilados hacia un sitio Web o una aplicación móvil. Para determinar la confiabilidad del sensor y del dispositivo en general se contrastan los valores capturados con valores referenciales obtenidos desde entidades dedicadas al monitoreo ambiental. El punto de partida de la investigación se centra en conocer el estado tecnológico actual de sistemas similares; a partir de aquello se hace énfasis en las carencias encontradas relacionadas a la exportación de los datos que estos sensores capturan; posteriormente se describen los procesos para la realización del sistema propuesto y sus respectivas evaluaciones. Los resultados del presente trabajo contribuyen a determinar la confiabilidad de este tipo de sistemas y, en general, amplía las opciones para futuros desarrollos relacionados a monitoreo medioambiental basado en loT.
\end{abstract}

Palabras clave: radiación ultravioleta, iot, arduino, raspberry pi.

\begin{abstract}
The present research work publishes the creation and evaluation of a low-cost system with Internet access for UV-B ultraviolet radiation measurement. The developed system uses hardware components such as Arduino boards, Raspberry Pi, light sensors, UV sensor; and software programming in languages such as $\mathrm{C}++_{+}$, Python, HTML and JavaScript. The hardware also has a wireless connection device that makes it easy to connect to the Internet to publish the collected data to a website or a mobile application. To determine the reliability of the sensor and the device in general, the captured values are contrasted with reference values obtained from entities dedicated to environmental monitoring. The starting point of the research focuses on knowing the current technological state of similar systems; from that, emphasis is placed on the gaps found related to the export of the data that these sensors capture; Subsequently, the processes for the realization of the proposed system and their respective evaluations are described. The results of the present work contribute to determine the reliability of this type of systems and, in general, expand the options for future developments related to environmental monitoring based on loT.
\end{abstract}

Keywords: ultraviolet radiation, uv, iot, arduino, raspberry pi.

Información del manuscrito:

Fecha de recepción: 21 de febrero de 2020

Fecha de aceptación: 20 de abril de 2020

Fecha de publicación: 05 de mayo de 2020 


\section{Introducción}

Uno de los efectos más frecuentes de las sobre exposición a la luz solar son las quemaduras 0 enrojecimiento de la piel. Esto se produce debido principalmente a la presencia de los rayos ultravioleta tipo B (UV-B) que son los que producen problemas de salud (Mora Ochoa, Olivares Savigñon, González Gross, \& Castro Mela, 2010). La excesiva exposición a este tipo de radiación UV-Bes uno de los principales agentes involucrados en la aparición de melanomas en el cuerpo humano, ya que podría modificar el ADN de los melanocitos y producir lesiones. El protector natural que la tierra dispone para minimizar los efectos de la radiación solar es la capa de ozono, por lo cual una debilitación en ella influirá en el aumento de problemas en la piel en la población (Becerra Mayor \& Aguilar Arjona, 2001).

El objetivo principal que se pretendió lograr en la investigación realizada es el de diseñar un prototipo funcional de hardware que obtenga valores acertados de la radiación ultravioleta UV-B captados por la incidencia directa del sol y mostrarlos en una pantalla acoplada al dispositivo y también en una página Web en el Internet. El presente trabajo de investigación pretende también ser un compendio de las pautas a seguir, así como de las herramientas básicas necesarias para la construcción del prototipo de hardware de medición de radiación UV-B utilizando componentes de bajo costo que permita obtener valores confiables de la medición a realizar. De forma general, entre la tecnología y arquitectura empleada destacan los principales componentes: sensor de rayos ultravioleta, sensores de luz tipo LDR, placas Arduino UNO, placa Raspberry Pi, Servidor Web y Aplicación móvil.

A lo largo del texto, primero se enfocan las áreas referentes a la problemática que resulta de la excesiva exposición a los rayos ultravioletas para el ser humano, ya que los daños provocados por estos excesos no son visibles al momento y eso ahonda más en la importancia de una adecuada protección (González-Púmariega, Tamayo, \& Sánchez-Lamar, 2009; Mora Ochoa et al., 2010). Se abarcan también aquella literatura existente relacionada con trabajos anteriores y 
con productos de similares características tanto con equipos profesionales como aquellos desarrollados muy acertadamente de forma independiente. Posterior se propone la utilización de hardware Arduino, Raspberry Pi y sensores UV junto con varias piezas de software com $\mathrm{C}++_{+}$, Python, JavaScript y HTML que interactúan todas entre ellas para poder realizar la respectiva captura de datos del lector y mostrarla en un servidor Web, todo sin intervención humana y de forma casi instantánea. Por último, se muestran los respectivos detalles comparativos resultantes de la investigación realizada junto con su correspondiente análisis, así como las obras que podrían derivarse a partir del presente trabajo.

\subsection{Importancia del problema}

Las radiaciones o rayos ultravioletas (UV) son radiaciones electromagnéticas no ionizantes que tienen una longitud de onda de entre $100 \mathrm{~nm}$ y $400 \mathrm{~nm}$. En pequeñas cantidades la exposición a este tipo de radiación solar es beneficiosa para el ser humano puesto que ayuda a producir vitamina D. Por el contrario, en grandes cantidades o sobre exposiciones pueden derivar en problemas serios de la piel, enfermedades oculares, quemaduras solares, entre otros (Mora Ochoa et al., 2010).

La radiación ultravioleta es un factor de luminosidad que se mide según ciertas especificaciones y se interpreta mejor con las recomendaciones dadas por organismos como la Organización Mundial de la Salud (OMS).En los actuales momentos la intensidad de la luz solar y el grupo de rayos que de él se desprenden suelen ser en ocasiones perjudiciales para la piel del ser humano, de allí que surge la necesidad de realizar el presente compromiso para plantear otras posibilidades de hacer más accesible tanto la información como las recomendaciones referentes a los niveles de radiación ultravioleta que afectan a la población.

En la siguiente tabla se ilustran los aspectos más relevantes de los rayos ultravioleta relacionados con su nivel de penetración en la 
atmósfera y su incidencia en la salud.

Tabla 1. Características de los Rayos ultravioleta.

\begin{tabular}{|c|c|c|c|c|}
\hline $\begin{array}{c}\text { Rayos } \\
\text { Ultravioleta }\end{array}$ & $\begin{array}{c}\text { Longitud } \\
\text { de Onda }\end{array}$ & $\begin{array}{c}\text { Penetración } \\
\text { en la } \\
\text { Atmósfera }\end{array}$ & $\begin{array}{c}\text { Relación } \\
\text { con la luz } \\
\text { solar } \\
\text { recibida }\end{array}$ & $\begin{array}{c}\text { Incidencia } \\
\text { en la salud }\end{array}$ \\
\hline $\begin{array}{c}\text { Ultravioleta } \\
\text { A }\end{array}$ & $\begin{array}{c}320- \\
400 \mathrm{~nm}\end{array}$ & $95.5 \%$ & $5.1 \%$ & Moderado \\
\hline $\begin{array}{c}\text { Ultravioleta } \\
\text { B }\end{array}$ & $\begin{array}{c}280- \\
320 \mathrm{~nm}\end{array}$ & $5.5 \%$ & $0.3 \%$ & Alto \\
\hline $\begin{array}{c}\text { Ultravioleta } \\
\text { C }\end{array}$ & $\begin{array}{c}100- \\
280 \mathrm{~nm}\end{array}$ & $0 \%$ & $0 \%$ & Muy Alto \\
\hline
\end{tabular}

Fuente: (González-Púmariega et al., 2009; Mora Ochoa et al., 2010)

Los rayos ultravioletas a pesar de que alcanzan la superficie de la tierra con muy poca incidencia, aproximadamente el $5 \%$ del total de la luz recibida, con relación a la luz visible son perjudiciales para el ser humano y en general para la vida en la tierra, por esto se hace importante tener una cultura de protección y prevención para evitar la sobre exposición a la luz solar especialmente en las horas de mayor incidencia del sol (GonzálezPúmariega et al., 2009).

Si bien la luz ultravioleta del tipo C resultaría ser la más dañina para la vida, nuestro planeta tiene la ventaja de contar con una barrera de protección que bloquea los rayos solares, sin embargo sus inmediatos sucesores, los rayos UV-B y UV-A, sí ingresan a la atmósfera y, aunque es muy poco su porcentaje de participación en relación con los demás que componen la luz solar, estos pueden ser causantes de severas lesiones a nivel de la piel e incluso a nivel interno en el organismo si no se toman las precauciones del caso en su debido momento. Además, muchos de estos problemas son de aparición tardía, es decir no son visibles inmediatamente, sino que suelen a veces presentar un efecto acumulativo que hace que los problemas de salud sean visibles mucho tiempo después, incluso años, de que las incidencias de la prolongada y repetitiva sobre exposición a la luz solar dañina se hizo presente en el cuerpo (González-Púmariega et al., 2009; Mora Ochoa et al., 2010). De acuerdo con algunos autores (Becerra Mayor \& Aguilar Arjona, 2001; González-Púmariega et al., 2009; Mora Ochoa et al., 2010; Organización Mundial de la Salud, 2003), la prevención que la población debe seguir para protegerse de los severos efectos que produce la sobre exposición a los rayos solares dañinos de la radiación ultravioleta UV-Bse basa 
principalmente en dos factores que dependen también de la educación de la población:

1) Prevención y protección solar

2) Preservar la capa de ozono

Sobre la Prevención y Protección

Solar se pueden tomar en cuenta los siguientes aspectos como primordiales (Mora Ochoa et al., 2010; Organización Mundial de la Salud, 2003):

- Reducir la exposición en horas centrales del día.

- Utilizar prendas de protección.

- Utilizar cremas de protección solar adecuadas con un factor de protección (FPS) 15+

- Utilizar sombreros y/o gafas que brinden la protección adecuada.

- Evitar las fuentes de bronceado artificial.

En base a lo anterior se hace necesario que la población pueda disponer de este tipo de información de la forma más ágil posible y acorde al lugar en que se necesite para empezar a hacer conciencia en cada individuo sobre los riesgos de la sobreexposición a la radiación solar y poder también tomar las medidas protectoras del caso. Al momento existen líneas de tecnologías bien diferenciadas en cuanto a sistemas o implementos de medición de la radiación solar: los sistemas comerciales que involucran un alto costo de adquisición e implementación, y los sistemas de bajo costo que son desarrollados en ocasiones como parte de experimentos 0 proyectos personales. En el mercado global se pueden encontrar varios tipos de dispositivos comerciales que permiten obtener valores ambientales sobre la intensidad de los rayos ultravioletas UV-B y/o UVA. Sus componentes y características varían entre los fabricantes. Se muestra a continuación una tabla comparativa de varios de los principales productos existentes en el mercado comercial:

Tabla 2. Algunos de los Productos comerciales existentes para medición UV.

\begin{tabular}{|l|c|c|c|c|}
\hline Modelo & Fabricante & $\begin{array}{c}\text { Rango de } \\
\text { operación }\end{array}$ & $\begin{array}{c}\text { Salida de } \\
\text { datos }\end{array}$ & $\begin{array}{c}\text { Precio } \\
\text { estimado }\end{array}$ \\
\hline $\begin{array}{l}\text { UVS-B- } \\
T\end{array}$ & $\begin{array}{c}\text { Kipp \& } \\
\text { Zonen }\end{array}$ & $\begin{array}{c}280-315 \\
\mathrm{~nm}\end{array}$ & $\begin{array}{c}\text { Analógica }+ \\
\text { conversor } \\
\text { externo }\end{array}$ & $\begin{array}{c}\$ 400 \\
0\end{array}$ \\
\hline $\begin{array}{l}\text { UVB } \\
\text { MS- } \\
212 \mathrm{~W}\end{array}$ & EKO & $280-315 \mathrm{~nm}$ & $\begin{array}{c}\text { Analógica }+ \\
\text { conversor }\end{array}$ & - \\
\hline
\end{tabular}

Fuente: Autores. 
Por otro lado, se pueden encontrar también soluciones experimentales que han intentado emular las características principales de los sistemas de medición UV profesionales. Se muestran a continuación algunos de estos desarrollos.

Tabla 3. Trabajos experimentales sobre Sistemas de medición UV.

\begin{tabular}{|c|c|c|c|}
\hline $\begin{array}{c}\text { Componentes } \\
\text { principales }\end{array}$ & $\begin{array}{l}\text { Rango de } \\
\text { operación }\end{array}$ & $\begin{array}{c}\text { Salida de } \\
\text { datos }\end{array}$ & Referencia \\
\hline $\begin{array}{l}\text { Fotodiodo PIN } \\
\text { OP910W }\end{array}$ & $\begin{array}{c}600- \\
1100 \mathrm{~nm}\end{array}$ & $\begin{array}{c}\text { No } \\
\text { documentado }\end{array}$ & $\begin{array}{c}\text { (Chacon } \\
\text { Cardona, Cely, \& } \\
\text { Guerrero, 2008) }\end{array}$ \\
\hline $\begin{array}{c}\text { Arduino UNO } \\
\text { Sensor SVT-UV-A } \\
\text { Amplificador } \\
\text { OPA2350UA }\end{array}$ & $\begin{array}{c}250-360 \\
\mathrm{~nm}\end{array}$ & Ethernet & $\begin{array}{c}\text { (Pérez Tiscareño, } \\
2014)\end{array}$ \\
\hline $\begin{array}{c}\text { Sensor } \\
\text { fotodiodo } \\
\text { UVD30A } \\
\text { Amplificador } \\
\text { AD620 Arduino } \\
\text { Nano }\end{array}$ & $\begin{array}{c}200-370 \\
n m\end{array}$ & $\begin{array}{c}\text { USB } \\
\text { Ethernet }\end{array}$ & $\begin{array}{l}\text { (Romero Gómez } \\
\& \text { Moreno } \\
\text { Roballo, 2016) }\end{array}$ \\
\hline
\end{tabular}

Fuente: Autores.

Al observar la documentación existente de ejemplos como los citados en las tablas 2 y 3 , se puede notar que el desarrollo de este tipo de hardware a nivel comercial y experimental se centra en brindar mayor exactitud y confiabilidad en las medidas que se obtienen de los sensores, pero carecen de información adecuada referente al cómo tratar los datos obtenidos, su exportación hacia un medio de almacenamiento y/o difusión para tareas investigativas o informativas.
También se merece especial atención el hecho muy particular de que se demuestra poco interés en la vinculación de este tipo de dispositivos hacia el Internet.

Si bien muchos de los trabajos experimentales analizados utilizan componentes que son relativamente sencillos de conectar al Internet, se hace muy poca mención sobre aquello en dichos documentos. $Y$ es que, como se mencionó en líneas anteriores, los autores de los trabajos analizados han dedicado especial atención en brindar la mayor exactitud posible en cuanto a sus mediciones, pero en cambio han relegado a segundo plano el hecho de la exportación automática de esos datos hacia el Internet para su posterior sometimiento a juicio, análisis 0 interpretación y divulgación.

Debido a lo anterior se hace necesario realizar las experiencias pertinentes que permitan establecer la justificación necesaria para el desarrollo profesional de herramientas para medir la incidencia directa de los rayos ultravioleta sobre la superficie terrestre utilizando componentes confiables y de bajo costo, y 
vinculadas hacia el Internet para la publicación e interpretación de los resultados obtenidos; todo esto mediante la creación de un prototipo funcional que utilice sensores de medición de radiación UV junto con el Internet para exportar los datos. Estos resultados obtenidos serán procesados para brindar información adecuada para la salud y cuidado de la piel de acuerdo con las mediciones obtenidas y según las recomendaciones de la OMS.

\section{Metodología.}

Para que el presente trabajo pueda llevarse a cabo sin dejar de lado la consecución final de los objetivos planteados al inicio de su elaboración, se decidió utilizar componentes tanto de hardware como de software que beneficien el alcance de las metas mencionadas, justificándose en parte las elecciones de los distintos componentes empleados.

De forma general, entre la tecnología y arquitectura empleada destacan los principales componentes: sensor de rayos ultravioleta, sensores de luz tipo LDR, placas Arduino UNO, placa Raspberry Pi, servidor Web, Base de datos, servicio Web y aplicación. En los siguientes párrafos se detallan las características principales tanto de los componentes de hardware como de software seleccionados en la elaboración del presente modelo tecnológico.

\subsection{Componentes principales de} Hardware

Figura 1. Esquema de hardware del proyecto realizado.

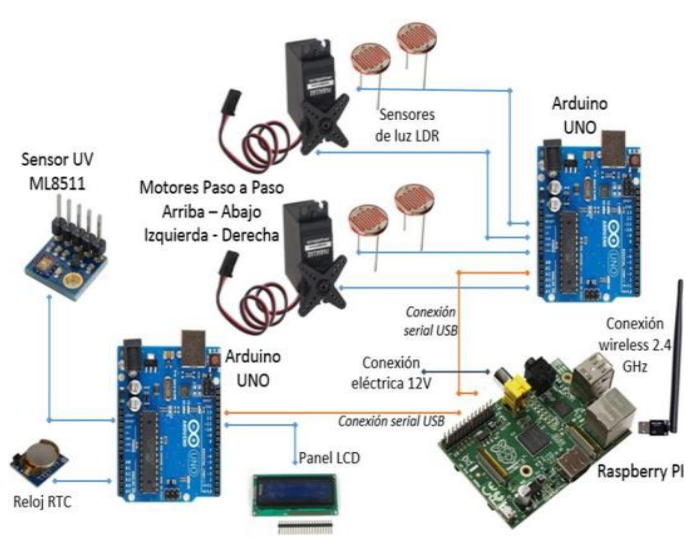

Fuente: Arduino Uno.

\subsection{Sensor de luz ultravioleta}

Dentro del mercado tecnológico existen distintos tipos de sensores de luz ultravioleta de relativa facilidad de implementación. Se resume a continuación aquellos que prestan mejores facilidades de adaptación con el hardware Arduino, que es el microcontrolador utilizado en el proyecto, y también aquellos que son relativamente más sencillos 
de conseguir en el mercado nacional:

Tabla 3. Características de sensores comerciales de Radiación UV para Arduino.

\begin{tabular}{|c|c|c|c|}
\hline Sensor & $\begin{array}{c}\text { Tipo de } \\
\text { salida }\end{array}$ & $\begin{array}{c}\text { Valores } \\
\text { de salida }\end{array}$ & $\begin{array}{c}\text { Longitud de } \\
\text { onda }\end{array}$ \\
\hline VEML6070 & Steps & $\begin{array}{c}0 \%- \\
100 \%\end{array}$ & $320 \mathrm{mn}-410 \mathrm{~nm}$ \\
\hline ML-8511 & Voltios (V) & $0 \mathrm{~V}-3.5 \mathrm{~V}$ & $280 \mathrm{~nm}-365 \mathrm{~nm}$ \\
\hline GUVA-S12SD & $\begin{array}{c}\text { Milivoltios } \\
(\mathrm{mv})\end{array}$ & $\begin{array}{c}0 \mathrm{mV}- \\
1200 \mathrm{mV}\end{array}$ & $240 \mathrm{~nm}-370 \mathrm{mn}$ \\
\hline
\end{tabular}

Fuente: Autores.

El sensor de rayos ultravioleta ML8511 es un sensor que tiene una salida de voltaje de 0V a 3.5V, posee además un amplificador interno que lo hace relativamente más sencillo de operar frente a otros de su tipo puesto que no requiere un conversor o amplificador externo para poder interpretar los datos. Dispone además de un rango de operación de longitud de onda que abarca todo el espectro UV-B que es la luz ultravioleta dañina y parte del espectro UV-A (LAPIS Semiconductor, 2013).

Su salida de voltaje operacional está linealmente relacionada con la intensidad UV en $\mathrm{mW} / \mathrm{cm} 2$.Esto significa que a un voltaje de ov le corresponde un índice UV 0 y a un voltaje de $3.0 \mathrm{~V}$ le corresponde un índice UV de 15 que es valor máximo que el sensor puede reportar en base a la escala de la OMS (Organización Mundial de la Salud, 2003, p. 6). Los valores UV intermedios son reportados como números decimales con una expresión de uno a dos decimales. Esta relación lineal permite que el sensor pueda ser operado directamente por un microcontrolador sin mayores complicaciones ni componentes adicionales para manipular la señal recibida.

Figura 2. Relación entre salida de Voltaje e Intensidad UV del sensor ML8511.

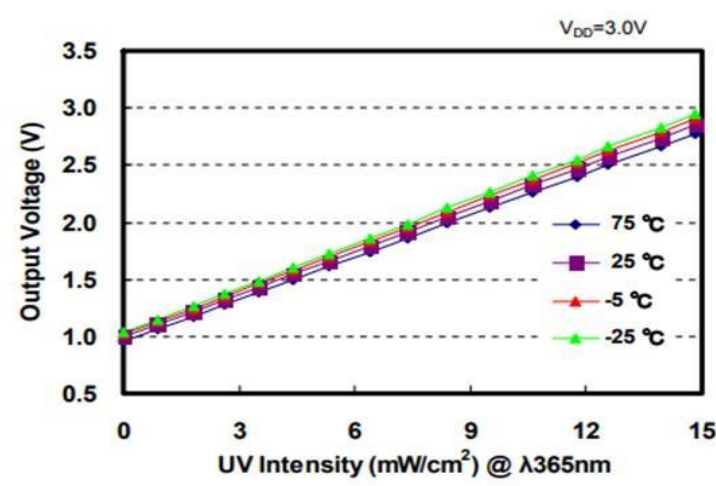

Fuente: (LAPIS Semiconductor, 2013).

Figura 3. Rango de operación de longitud de onda del Sensor UV ML8511.

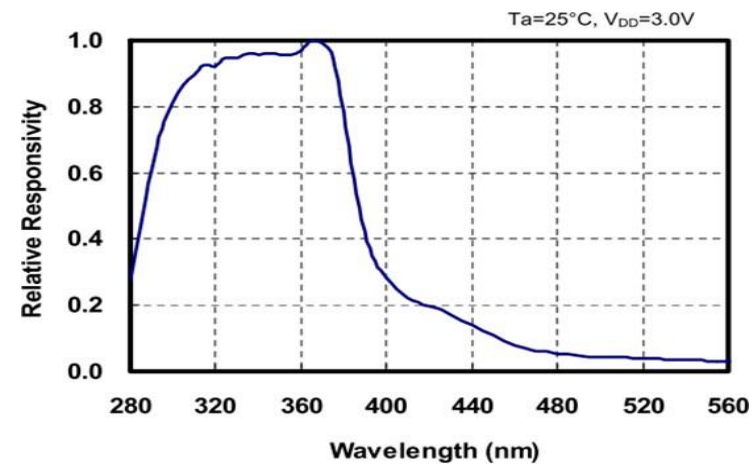

Fuente: (LAPIS Semiconductor, 2013). 
Para el presente proyecto se elige el sensor ML8511 frente a otros debido a su sencillez de implementación, disponibilidad y relativa facilidad de conversión del voltaje recibido a valores del Índice UV de la OMS.

\subsection{Fotorresistencias y motores}

El presente proyecto incorpora un sistema diseñado específicamente para él que consiste en un "Sistema de seguimiento solar" que reúne cuatro (4) sensores de luz tipo LDR (o) también llamados fotorresistencias) distribuidos convenientemente rodeando el sensor UV para que permitan que en todo momento el sensor ultravioleta esté direccionado hacia el sol, haciéndolo girar hacia él a través de dos motores adjuntos. Esto es una parte importante del proyecto puesto que una de las limitantes de los sensores UV comerciales es su muy bajo rango de captación de luz solar (LAPIS Semiconductor, 2013) y por tal motivo estos sensores deben estar siempre direccionados correctamente hacia el disco solar para que puedan obtener lecturas acertadas.

\subsection{Arduino}

Las placas de hardware Arduino son plataformas electrónicas diseñadas y pensadas para un uso fácil tanto del hardware como de software. Los tableros electrónicos Arduino son capaces de leer entradas de distintos dispositivos electrónicos como: sensores de humedad, calor, etc., pulsaciones de botones, y con esto ejecutar una determinada acción como: encender/apagar un led, enviar un mensaje a otro dispositivo, etc. Para el presente proyecto se eligieron dos placas Arduino UNO debido a que su poder de procesamiento (Flash Memory 32KB y Clock Speed $16 \mathrm{MHz}$ ) y cantidad de entradas analógicas y digitales (6 $\begin{array}{llll}\text { entradas analógicas y } & 6\end{array}$ entradas/salidas digitales) (Arduino AG, 2018) resultaron ser suficientes para aquello que se pretendía desarrollar.

\subsection{Raspberry Pi}

Existen varios tipos de circuitos 0 placas electrónicas que pueden utilizarse como dispositivos de base para proyectos basados en Internet de las Cosas. Entre las características de éstos se puede 
mencionar aspectos como: Sistema Operativo, memoria RAM, procesador, puertos de comunicación, flexibilidad en cuanto a lenguajes de programación, etc. De acuerdo con las comparaciones realizadas por Maksimović, et al. (Maksimović, Vujović, Davidović, \& Perišić, 2014) las placas Raspberry PI poseen especificaciones técnicas que las convierten en una buena opción para servir de puente de comunicación entre distintos tipos de componentes y periféricos. Los dispositivos Raspberry PI son placas de circuitos de poco tamaño y con características de memoria y procesamiento tal que permiten ejecutar un Sistema Operativo y hacer que dichos componentes se comporten como un mini computador. Estos dispositivos vienen con un único chip que contiene la memoria, unidad central de procesamiento y chip gráfico. Las placas Raspberry PI usan chips ARM similares a los usados en varios smartphones y tablets del mercado, pero con la diferencia de que los usados en el Raspberry PI son un poco más lentos pero lo suficientemente rápidos para el trabajo que requieren (Raspberry PI, 2015).
La placa Raspberry PI en el presente proyecto cumple la función de ser el centro principal de procesamiento y comunicación. Debido a las ventajas que brinda esta placa electrónica al comportarse como un computador, se la elije para trabajar a la par de las placas Arduino y sirviéndoles a éstas como el receptor de la comunicación y el encargado de exportar los datos hacia el Internet. El Sistema Operativo que se instala sobre dicha placa es Raspbian debido a que es una versión de Debian, Sistema Operativo GNU/Linux, muy completa pero adaptada para la arquitectura de los dispositivos Raspberry PI, lo cual permite que se obtenga el máximo provecho de estos (Harrington,2015).

De entre los distintos modelos de placas Raspberry $\mathrm{PI}$ se elige el modelo $\mathrm{B}+$ debido a que posee incorporado un puerto Ethernet y cuatro puertos USB que son suficientes para la comunicación entre los distintos dispositivos que se requieren para el proyecto.

\subsection{Alimentación de energía}

A través de la conexión USB la placa Raspberry Pi alimenta de energía a los microcontroladores Arduino y estos a su vez proveen energía a los 
distintos motores, sensores, pantalla LCD y demás componentes adjuntos. El dispositivo Raspberry Pi se alimenta de energía eléctrica mediante su propio adaptador de corriente que transforma la corriente alterna recibida (110V en la región local) y la transforma en 5V (V: Voltios) y 2.5A (A: Amperios) de corriente continua. En esa transformación de corriente, el dispositivo empleado (Raspberry $\mathrm{Pi}$ modelo $\mathrm{B}+$ ) varía su consumo energético según las tareas y cargas de trabajo que deba realizar. Así dicho consumo varía desde los 360mA hasta los 480mA (mA: miliamperios) en funcionamiento completo.

Por otro lado, el microcontrolador Arduino UNO tiene una alimentación típica de energía de $5 \mathrm{~V}$ y un consumo energético que ronda los 200mAh (mAh: miliamperios hora) para un proyecto de relativa poca carga como los realizados en el presente trabajo.

En caso de requerirse dotar de autonomía energética al proyecto entero resulta suficiente proveer de bancos de baterías de buena capacidad de los muchos que existen en el mercado comercial. Estos dispositivos poseen capacidades de energía variadas según las necesidades y pueden rondar los $30000 \mathrm{mAh}$, lo cual resulta ser suficiente para alimentar de energía al sistema entero por uno a dos días dependiendo la carga de trabajo que tengan.

Para el presente proyecto, la energía de alimentación de los dispositivos vinculados fue obtenida directamente de las tomas eléctricas cercanas a los lugares de pruebas, sin embargo, el diseño y la naturaleza de los componentes empleados brindan la posibilidad de obtener autonomía energética por un período de tiempo considerablemente suficiente para realizar pruebas y mediciones sin tener que recargar o cambiar las baterías utilizadas.

\subsection{Conexión de datos}

El envío de los datos capturados por el sensor desde la placa Arduino hacia el Raspberry $\mathrm{Pi}$ se efectúa mediante una conexión Serial USB entre ambos dispositivos. La conexión a Internet desde el Raspberry $\mathrm{Pi}$ se realiza utilizando componentes de hardware 
compatibles con Sistemas Operativos basados en Linux, principalmente aquellos que incorporan en su arquitectura de hardware chipset RealTek.

Estos dispositivos wifi son sencillos y económicos de conseguir en el mercado tecnológico local. Estos utilizan como estándar de comunicación inalámbrica el IEEE $802.11 \mathrm{~b} / \mathrm{g} / \mathrm{n}$ lo cual significa una comunicación wifi en la banda de los 2.4GHz con velocidades de hasta 300 Mbps de velocidad de conexión inalámbrica en base al estándar IEEE 802.11 ny con una velocidad de transmisión real que ronda los $150 \mathrm{Mbps}$ lo cual resulta más que suficiente para el envío y recepción de información desde el dispositivo Raspberry Pi hacia el servicio web del servidor de Internet.

\subsection{Componentes de Software}

Figura 3. Componentes de software.

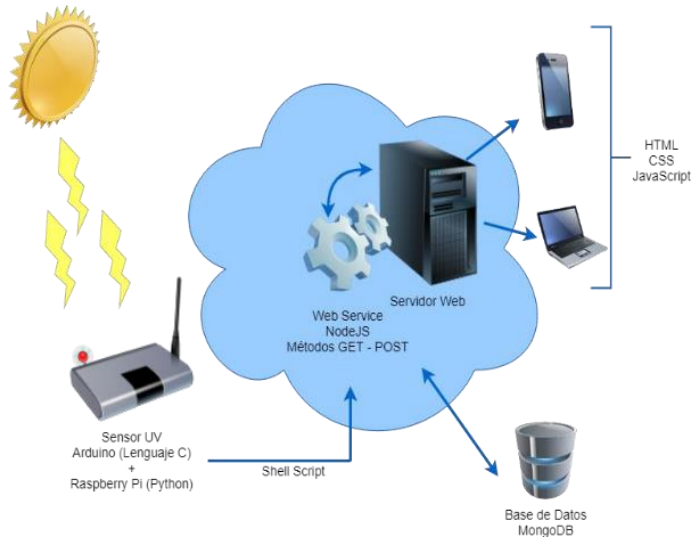

Fuente: Autores.

\subsection{Sensor UV y Arduino}

El desarrollo de la programación en la placa Arduino consiste en ajustar los niveles de medida del sensor para obtener los voltajes del sensor y convertirlos a valores de irradiancia en $\mathrm{mW} / \mathrm{cm} 2$ y a valores UV (índice UV según la OMS). Esta programación recibe lecturas constantes del sensor UV y realiza los cálculos y conversiones adecuadas para mostrar el valor de irradiancia y el índice UV promediado de 8 lecturas consecutivas cada 1 segundo.

La programación o código fuente de la placa Arduino y su sensor UV está realizada en Lenguaje $C$ y permite la comunicación desde el Arduino hacia el sensor UV y desde el Arduino hacia Raspberry Pi.

\subsection{Raspberry Pi como puente} de comunicación entre

\section{Arduino y el Internet}

La programación en el dispositivo Raspberry está destinada principalmente a administrar la comunicación tanto hacia el dispositivo Arduino como hacia el Internet y básicamente se enfoca en los siguientes puntos: 
- Comunicación Raspberry Pi Arduino: Obtiene los valores del sensor UV por medio de la comunicación serial entre ambos dispositivos. Está desarrollado en Python y permite leer datos UV proporcionados por el Arduino en uno de puertos de comunicación del Raspberry Pi.

- Comunicación Raspberry Pi Internet: Esta comunicación está realizada en el lenguaje de programación Shell Script de Linux. Esto significa que mediante un script desde línea de comandos se envían las peticiones al método Post del Web Service del Servidor HTTP de la aplicación.

Los datos que se envían desde el Raspberry PI hacia el Web Service de Internet son los siguientes:

- Identificación del dispositivo (ID, nombre)

- Ubicación (localidad, dirección)

- Valores del sensor (índice UV, hora, fecha) $\leftarrow$ Campo Índice UV puede variar en función del tipo de sensor

- Tipo de sensor (UV, ambiente, temperatura, polución, etc.)
Debido a la naturaleza de la información procesada y la importancia de su adecuada divulgación, se utiliza un servidor central basado en servicios en la nube. Éste almacena los datos recopilados desde el dispositivo Raspberry $\mathrm{Pi}$ en una instancia de una base de datos para crear registros de medidas históricos, publica servicio web para poder consultar información del sensor o ingresar nuevos datos de lecturas. El servidor en la nube está destinado para almacenar y/o mostrar la siguiente información:

- Identificación de dispositivo (ID, nombre)

- Ubicación (Localidad, dirección)

- Valores del sensor (índice UV, hora, fecha)

- Tipo de sensor (UV, ambiente, temperatura, polución, etc.)

- Recomendaciones

- Histórico por horas, días, semanas, etc.

El Servicio Web contenido en el servidor de Internet está desarrollado en NodeJS y proporciona los métodos básicos de GET y POST para que sean 
accesibles desde el dispositivo sensor creado. En el diseño no se contempla el método DELETE de forma pública debido a que los sensores no están en capacidad de auto corregirse si existiese una anomalía en el envío de datos.

\section{Resultados y discusión.}

Debido a que en el Ecuador, al momento de realizar las pruebas respectivas, solamente se contaba con información de la incidencia de los rayos ultravioleta en las dos ciudades principales del país que son Quito y Guayaquil, los datos tomados como referencia para el presente trabajo fueron proporcionados por el sitio Web de la Agencia Espacial Civil Ecuatoriana (www.exa.ec),la cual es una institución sin fines de lucro encargada, entre otras actividades, de informar a la ciudadanía sobre la incidencia de los rayos ultravioleta en el Ecuador tomando como referencia la ciudad de Guayaquil ya que es allí donde alojan sus oficinas $y$ red de monitoreo ambiental. Al momento de obtener los valores UV esta Agencia disponía de sistemas de medición de muy altas prestaciones, lo cual garantizaba un ínfimo error en sus mediciones y servían como punto de referencia para establecer el nivel de veracidad del sistema de medición UV desarrollado.

Las medidas de prueba realizadas con el dispositivo experimental fueron obtenidas durante un lapso de doce horas en la ciudad de Guayaquil en las primeras semanas del mes de agosto del año 2017 y teniendo en cuenta siempre que se cumplan las siguientes condiciones para la toma de las muestras:

- A la intemperie.

- Sin ningún tipo de protección o cubierta plástica o de cristal para el sensor UV.

- En presencia de un cielo claro, despejado y sin nubes.

- En un espacio con visibilidad directa a, por lo menos, una parte del recorrido solar.

El sitio web de la Agencia Espacial Civil Ecuatoriana en su momento no ofrecía las condiciones necesarias para realizar las adecuadas consultas o descargas de registros históricos de las lecturas tomadas por sus dispositivos. La página web que muestra los datos de sus sensores (http://guayaquil.exa.ec) era una página web con contenido 
estático que se actualizaba automáticamente cada 5 minutos para reescribir los valores de sus sensores. En base a lo anterior se desarrolló un script en Bash Linux para que obtenga los valores del sitio web cada 5 minutos y los almacene en un archivo plano en una ubicación accesible para su posterior consulta y comparación.

Los datos obtenidos a partir de las fuentes principales (Página Web del Sitio de la Agencia Espacial Civil Ecuatoriana http://guayaquil.exa.ec y modelo experimental realizado) fueron organizados, para su publicación en el presente documento, en base a los horarios de toma de la muestra.

Debido a que la incidencia del sol es mayor a partir de las horas centrales del día, las muestras recolectadas (tanto de la Agencia Espacial Civil Ecuatoriana como del experimento realizado) fueron sesgadas durante un rango de tiempo que va desde las 10:00AM hasta las 16:00PMcon un intervalo de 5 minutos entre cada medida.

En las siguientes figuras se ilustra la comparación de los datos obtenidos desde la Agencia Espacial Civil Ecuatoriana y el modelo tecnológico desarrollado durante un lapso de dos horas (desde las 10:00AM hasta la 12:00PM, desde las 12:00PM hasta la 14:00PMy desde las 14:00PM hasta la 16:00PM) con un intervalo entre cada medición de 5 minutos.

Figura 5. Comparación de lecturas UV (II)

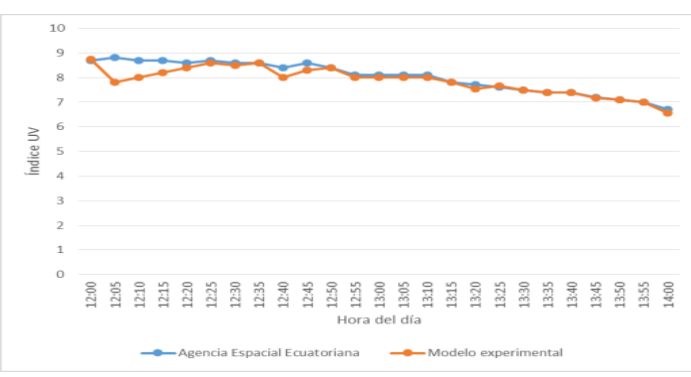

Fuente: Agencia Espacial Civil Ecuatoriana.

Figura 6. Comparación de lecturas UV (I)

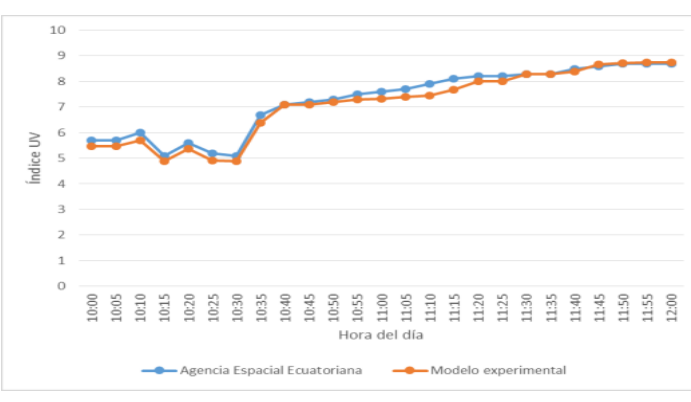

Fuente: Agencia Espacial Civil Ecuatoriana.

Figura 7. Comparación de lecturas UV (III)

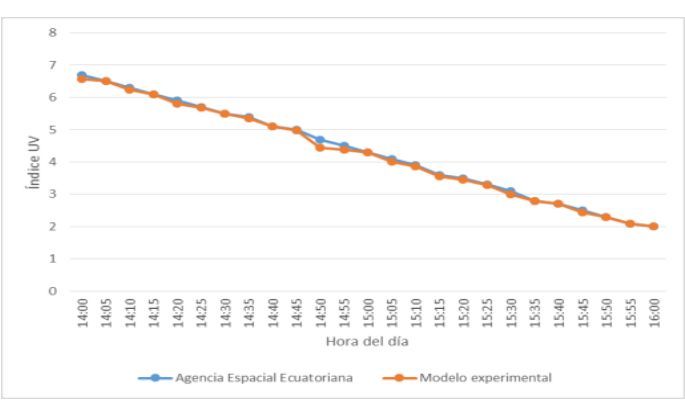

Fuente: Agencia Espacial Civil Ecuatoriana. 
Se muestran a continuación una comparativa en un día de cielo parcialmente nublado y durante un corto lapso.

Figura 8. Comparación de lecturas UV anómalas

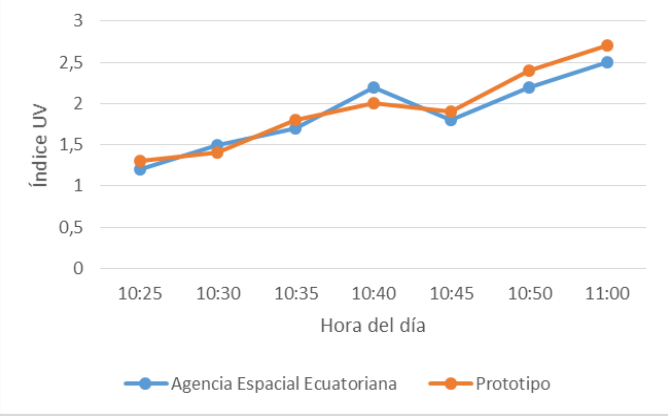

Fuente: Agencia Espacial Civil Ecuatoriana.

\subsection{Costos del proyecto}

A modo de referencia, se muestra a continuación un breve y sencillo resumen de los costos del hardware utilizado en el desarrollo del presente proyecto:

- Raspberry Pi B+ $=\$ 50$

- Arduino $\mathrm{UNO}(\mathrm{x} 2)=\$ 30$

- Motores (x2) $=\$ 20$

- Sensor UV ML8511 = $\$ 20$

- Sensores LDR $(x 4)=\$ 10$

- Panel LCD $=\$ 10$

- Clock RTC $=\$ 5$

- Antena wifi $=\$ 20$

- Acoples y extras $=\$ 20$

Costo total del hardware del proyecto $=\$ 185$.
Las lecturas de los datos obtenidos reflejan cierto comportamiento parecido con otros trabajos en los que también se emplearon equipamiento de hardware de bajo costo (Pérez Tiscareño, 2014; Romero Gómez \& Moreno Roballo, 2016) con lo cual se contrasta también la veracidad de la información que el sensor (y su adecuado montaje) proporcionan.

Tal como refleja en las especificaciones técnicas del sensor UV empleado (LAPIS Semiconductor, 2013) este tipo de sensores demuestran tener la ventaja de que al poseer un amplificador interno pasan a ser relativamente fáciles de utilizar en los proyectos de hardware y su programación al interior de la placa microcontroladora (Arduino en el caso del presente proyecto) se vuelve también sencilla de implementar.

Los datos recopilados demuestran también que este tipo de sensores son bastante sensibles y susceptibles a las interferencias en el disco solar ocasionadas principalmente por la presencia de nubes a su alrededor. El prototipo desarrollado si bien capta lecturas bastante aceptables al medir la 
incidencia directa del sol, en presencia de nubes u obstáculos de visón hacia el sol las lecturas se vuelven inexactas e incluso nulas mientras dura la presencia del obstáculo.

El sistema de seguimiento solar desarrollado para el mejor funcionamiento en cuanto a la captación de los datos de incidencia del sol demostró ser eficiente para que el módulo que contiene el sensor UV se ubique constantemente en posición de visibilidad directa al sol. Sin embargo, en las pruebas desarrolladas existieron ciertas anomalías que provocaron que en ocasiones los motores y rotores (que deben girar hacia el sol) se muevan innecesariamente provocando que las lecturas en ese corto tiempo no sean adecuadas.

En cuanto a las comparativas de la funcionalidad del componente de software frente a versiones comerciales se pueden resumir en las siguientes como las más importantes: el software desarrollado brinda la facilidad de exportar automáticamente los datos obtenidos hacia el Internet mientras que los dispositivos comerciales y profesionales requieren intervención física o adquirir módulos adicionales para esto, o incluso no lo incorporan en algunos modelos (Kipp \& Zonen, 2017; Solar Light, 2013). Adicional al software del propio prototipo desarrollado, se brinda la opción de que los datos puedan ser consultados directamente en el sitio web del prototipo o bien desde el teléfono celular, situación que los dispositivos comerciales no la brindad o en todo caso no hacen publicación de aquello en sus especificaciones.

\section{Conclusiones}

El presente desarrollo tecnológico permite a sus usuarios el obtener los datos de la radiación ultravioleta UVB y UVA en cielos claros, despejados y sin nubes; y con valores muy cercanos a la realidad, además dicha información recabada es también automáticamente exportada hacia el Internet mediante un Web Service para que pueda ser tratada y/o reproducida hacia otros destinos y pueda servir para lo que realmente se pretende: informar y prevenir a la ciudadanía en general de los riesgos que provoca la sobre 
exposición a los rayos ultravioleta dañinos.

Como conclusiones del presente trabajo de investigación se plasman las siguientes:

- El piloto funcional de hardware desarrollado permite medir acertadamente las incidencias directas de los rayos ultravioleta captados mediante un sensor UV y mostrarlos valores y alertas que corresponden de acuerdo con las normas especificadas por la Organización Mundial de la Salud.

- El hardware empleado para medir la luz ultravioleta del sol es un sensor UV relativamente sencillo de adquirir y de bajo coste que demostró en las pruebas ser completamente funcional siempre y cuando la incidencia del sol sea directa al sensor y sin obstáculos (edificaciones, nubosidad, etc.).

- La aplicación web para el piloto desarrollado demostró ser funcional al cumplir con los requisitos principales para los que fue elaborada.

- El Web Service de todo el sistema desarrollado demostró ser el eje principal de todo el proyecto a nivel de software. Sirve de conexión principal entre el hardware y la base de datos, entre la base de datos y la aplicación web y entre la base de datos y la aplicación móvil.

\section{Bibliografía.}

Arduino AG. (2018). Arduino Uno Rev3. Retrieved August 27, 2017, from https://store.arduino.cc/usa/ard uino-uno-rev3

Becerra Mayor, M. del mar, \& Aguilar Arjona, J. A. (2001). Radiobiología Revista electrónica Radiación ultravioleta y cáncer de piel. Consejos preventivos Radiación ultravioleta y cáncer de piel. Consejos preventivos. RB1pdf Radiobiología Radiobiología, 1(1), 15-17. Retrieved from http://wwwrayos.medicina.uma.es/rmf/rad iobiologia/revista/radiobiologia. htm

Chacon Cardona, C. A., Cely, O. E., \& Guerrero, F. (2008). Diseño y construcción de un medidor de radiación solar. Tecnura, 1423. 
González-Púmariega, M., Tamayo, M. V., \& Sánchez-Lamar, Á. (2009). LA RADIACIÓN ULTRAVIOLETA. SU EFECTO DAÑINO Y CONSECUENCIAS PARA LA SALUD HUMANA, 18(2), 69-80. Retrieved from http://www.ubiobio.cl/miweb/w ebfile/media/194/v/v18-

2/06.pdf

Harrington, W. (2015). Learning Raspbian. Retrieved from https://books.google.com/book $\mathrm{s} ? \mathrm{hl}=\mathrm{en} \& \mathrm{l}=\& \mathrm{id}=\mathrm{O} 6 \mathrm{HNBgAAQ}$ BAJ\&oi=fnd\&pg=PP1\&dq=rasp bian\&ots=ZG2PnhATWF\&sig= 0QwaUR3tOkvYgRWReEo5sg esoHo

Kipp \& Zonen. (2017). Kipp \& Zonen. Retrieved from http://www.kippzonen.es/Produ ct/248/UVS-B-T-RadiometroUV\#.WazPMMtSDIU

LAPIS Semiconductor. (2013). ML8511 UV Sensor with Voltage Output.

Maksimović, M., Vujović, V., Davidović, N., \& Perišić, V. M. and B. (2014). Raspberry Pi as Internet of Things hardware: Performances and Constraints.
ResearchGate, 3. Retrieved fromhttps://www.researchgate. net/profile/Vladimir_Vujovic/pu blication/280344140_ELI16_M aksimovic_Vujovic_Davidovic_ Milosevic_Perisic/links/55b336 8608ae9289a08594aa.pdf Mora Ochoa, M., Olivares Savigñon, R., González Gross, M., \& Castro Mela, I. (2010). El sol: ¿enemigo de nuestra piel? Medisan, 14(6), 825-837. Retrieved from http://scielo.sld.cu/scielo.php?s cript=sci_arttext\&pid=S102930192010000600014\&lang=es Organización Mundial de la Salud. (2003). Indice UV Solar mundial: guía práctica. Retrieved from http://www.who.int/uv

Pérez Tiscareño, M. (2014). Diseño de un radiómetro ultravioleta, para su aplicación en modelos de radiación UV, 76.

Raspberry PI. (2015). Ultimate guide to Raspberry PI. Computer Shopper, (324), 105-119. Retrieved from http://micklord.com/foru/Rasp 
berry Pi Pages from Computer

Shopper 2015-02.pdf

Romero Gómez, J. P., \& Moreno

Roballo, N. I. (2016).

IMPLEMENTACIÓN DE UNA

ESTACIÓN RADIOMÉTRICA

EN LA UNIVERSIDAD

DISTRITAL FRANCISCO

JOSÉ DE CALDAS.

Solar Light. (2013). Solar Light.

Retrieved from

http://solarlight.com/product/u

v-warning-signal/ 